\title{
An Alignment Method for time sequence Myocardial Perfusion MR image
}

\author{
Qi Zong \\ School of Electronic Information and Electrical Engineering \\ Shanghai Jiao Tong University \\ Shanghai,China \\ juexin@sjtu.edu.cn
}

\author{
Xin Yang \\ School of Electronic Information and Electrical Engineering \\ Shanghai Jiao Tong University \\ Shanghai, China \\ yangxin@sjtu.edu.cn
}

\begin{abstract}
MRI (Magnetic Resonance Imaging) of the myocardial perfusion plays an important role in the diagnosis of heart disease. However, it is important to alignment the timeseries MRI perfusion images before to detect the grey level change at correspondence point. In this paper, a new alignment method is presented which combinesprincipal axes with block matching method. This is a two-step strategy from rough to fine alignment. At the first step, as to the rough alignment, principal axes method is used to obtain the approximate displacement and rotation angle. At the second step, block matching method with SAD principle is used to achieve fine alignment of ROI(region of interest), which divides the referred image and the image to be registered into several small pieces and uses full search method to find the matched parts. As a result, the accuracy and computational speed of the non-rigid alignment is improved significantly.
\end{abstract}

Keywords-myocardial perfusion MR image; principal axes; block matching; non-rigid alignment.

\section{INTRODUCTION}

Myocardial perfusion imaging is a common diagnostic technique for coronary artery disease, which plays a very important role in the diagnosis of coronary artery disease ${ }^{[1]}$. Through it we can observe the changes of cardiac blood flow, and it can help to determine the specific position and range of obstructive myocardial ischemia and infarction. When the contrast medium is injected into the body, the brightness of nuclear magnetic resonance image sequences will change with time. The change of the intensity of MRI sequence can help the doctor make moreaccurate diagnosis ${ }^{[2]}$.

After obtaining myocardial perfusion image sequences, we need to make a statistical analysis of gray change of the corresponding points and establish the mathematical model to determine the relevant parameters. So we have to make sure of the space alignment of pixels at different times, especially the ones which can accurately reflect the patient's physiological state, such as the left ventricle related parts, in order to get access to the required information. Because it spends a rather long time of examining patients to get the perfusion images, they will inevitably appear breathing, heart beating or slight movement of the body, resulting in space differences in the $\mathrm{ROI}($ such as cardiac short axis sections) of MRI. These

This work was financially supported by National Key Basic Research and Development Plan (2010CB732506), Shanghai basic Science research found 12JC1406600. interference factors can bring some difficulties for the diagnosis using myocardial perfusion images.As a result, it is necessary to register the image sequences, so that the corresponding points of each frame are consistent in the space position, and it is the prerequisite for the follow-up medical image processing ${ }^{[3]}$.

Image alignment technology can be divided into two categories: rigid alignment and non -rigid alignment. Rigid alignment is assumed that the deformation of the object is rigid and there is only translation and rotation between images.At present, the research on the non-rigid alignment algorithm is mature, but there is no general algorithm for the non-rigid alignment for all the problems. However, in the actual clinical diagnosis of medical treatment, the deformation of some organs is generally not rigid, and the traditional methods are no longer appropriate. Luckily, non-rigid alignment can effectively improve the non-rigid deformation of the organs. Some non-rigid algorithms have been applied in the medical image alignment, resulting in relatively satisfactory results, such as the optical method ${ }^{[5-6]}$, mutual information method ${ }^{[7]}$ and Markoff random field method ${ }^{[4]}$. In this paper, a new method for non-rigid alignment is present.

\section{THE OVERALL ALGORITHM DESIGN OF MYOCARDIAL PERFUSION IMAGE ALIGNMENT}

In this paper, a method based on principal axes and block matching is proposed for the alignment of myocardial perfusion image sequences. The principal axes method, with small calculation, can be used for rough alignment of the sequence images, get a coarse result. Then, the method of block matching is used to perform fine alignment of the image, in order to improve the effect of alignment ${ }^{[7]}$.

The whole algorithm flow as shown in Fig.1: 


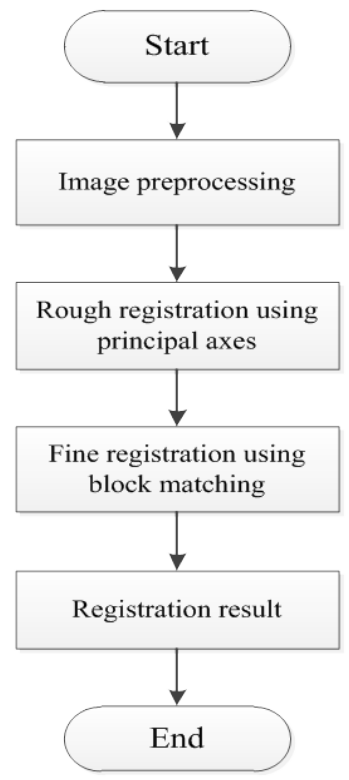

Fig.1.The whole algorithm flow

\section{BASIC THEORY OF MOMENT PRINCIPAL AXES ALIGNMENT METHOD}

The principal axes method was present by A Kak and Rosenfeld in 1982, and was first used in medical image alignment by Alpert in $1990^{[10-12]}$. The basic principle is to calculate the centroid and axis of the two images by using the principle of classical mechanics mass distribution, and then achieve the alignment of the two images by translation and rotation. Based on principal axes method calculation process: find the centroids of the images and then through the second order moment of the image to find angle image of the spindle and the coordinate system, and according to the two characteristics of image alignment ${ }^{[8-9]}$.

\section{A. Basic theory of moment principal axes}

The medical image alignment is mainly related to three parameters: horizontal movementx, vertical displacementy, and rotation angle theta $\theta$. The regional moment represents a normalized gray level image function as a probability density of 2 dimensional random variables. The moment of $(p+q)$ order depends onthe transformation of the scale, translation, rotation and even gray levels. And it is given by the following formula:

$$
m_{p q}=\iint_{-\infty}^{\infty} x^{p} y^{q} f(x, y) d x d y(1)
$$

$x_{c}$ and $y_{c}$ are the coordinates of center of gravity, which can be obtained by the following relations:

$$
x_{c}=\frac{m_{10}}{m_{00}} y_{c}=\frac{m_{01}}{m_{00}}(2)
$$

We can also obtain the $(\mathrm{p}+\mathrm{q})$ order center moment from the image, which has the characteristics of shift invariance, and is given by the following:

$$
\mu_{p q}=\iint_{-\infty}^{\infty}\left(x-x_{c}\right)^{p}\left(y-y_{c}\right)^{q} f(x, y) d x d y(3)
$$

The angle between the main axis of the image axis and the reference frame of the $\mathrm{Y}$ axis can be obtained by the two center moment:

$$
\theta=\frac{1}{2} \tan ^{-1}\left(\frac{2 \mu_{11}}{\mu_{20}-\mu_{02}}\right)(4)
$$

Assuming the coordinate transformation between the images is a rigid transformation, the expression is as follows:

$$
\left[\begin{array}{l}
x^{\prime} \\
y^{\prime}
\end{array}\right]=k\left[\begin{array}{cc}
\cos \theta & \sin \theta \\
-\sin \theta & \cos \theta
\end{array}\right]\left[\begin{array}{l}
x \\
y
\end{array}\right]+\left[\begin{array}{l}
\Delta x \\
\Delta y
\end{array}\right]
$$

The parameters ofk $, \theta, \Delta x, \Delta y$ the alignment parameters of two images ${ }^{[13]}$. Through(2) and (4),we can get $\theta$, $\Delta x, \Delta y$. The size of the image sequence in the experiment is not scaled,sok takes 1 .

Theprincipal axes is automatic, fast and easy to implement, so it can be applied widely in the field of the accuracy of alignment.Through the analysis of the principal axes method, we find the computational complexity of $n$ $\left(M^{*} N\right)$, where $\mathrm{n}$ is the first order moment and the two order moment, $M^{*} N$ is the image $\operatorname{size}(\mathrm{M}=192, \mathrm{~N}=144)$. Compared with the alignment method based on the feature and gray statistic, the principal axes method of the spindle has a faster operation speed.

\section{B. Principal axes method for rough alignment experiment}

The front frame image is used as the reference image, and the rear one is used as target image. After executing related program, the results shown in Fig.2 and Table.1:

\section{The first frame $F_{1}$ The second frame $F_{2}$}
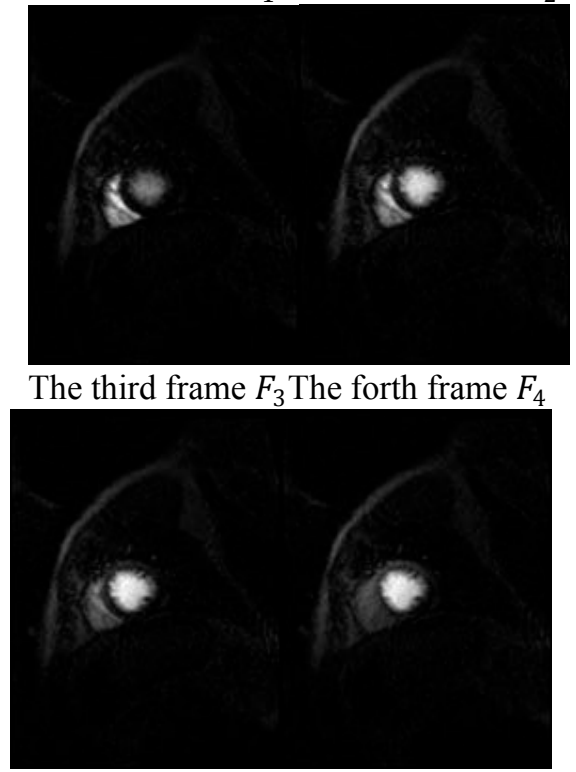

Fig.2 Four magnetic resonance images of myocardial perfusion

Table.1.The alignment results of principal axes method

\begin{tabular}{|l|l|l|l|}
\hline $\begin{array}{l}\text { Pairsof } \\
\text { registrated } \\
\text { images }\end{array}$ & $\Delta \mathbf{x} /$ pixel & $\Delta \mathbf{y} /$ pixel & $\begin{array}{c}\Delta \boldsymbol{\theta} \\
\left({ }^{\circ}\right)\end{array}$ \\
\hline $\boldsymbol{F}_{\mathbf{2}}-\boldsymbol{F}_{\mathbf{1}}$ & 0.13 & -0.57 & 0.73 \\
\hline $\boldsymbol{F}_{\mathbf{3}}-\boldsymbol{F}_{\mathbf{2}}$ & 0.72 & 0.17 & -2.15 \\
\hline $\boldsymbol{F}_{\mathbf{4}}-\boldsymbol{F}_{\mathbf{3}}$ & 0.14 & -0.12 & 0.69 \\
\hline
\end{tabular}

The calculation is small, and the speed is fast, which 
cannot reach the effect of alignment. But it can improve the accuracy of the next step fine alignment.

\section{USING BLOCK MATCHING FOR FINE ALIGNMENT}

According to the statistical characteristics of image, for myocardial perfusion image sequences, because of the very short time interval between two adjacent frames, it is almost similar between them ${ }^{[14]}$. Namely, the correlation between them is very strong. Due to the frame correlation, we can make block matching of a pair of adjacent frames and then estimate the motion vector between frames. When the small parts of images of the floating imagecorrected from the inverse motion vector, the images have been non-rigid aligned.

Block matching algorithm is a common method in computer vision and other research fields. It is usually used for motion compensation ${ }^{[17]}$ and video compression for video images $^{[15]}$. However, the alignment parameters of the image can be obtained by the algorithm of local matching sub image block, and the method can also improve the robustness of rigid alignment and realize non-rigid alignment. Find the corresponding block in the reference frame, and the prediction error, the location of the block in the current frame is obtained.All the blocks are operated in this way, and the final prediction frame image is the alignment image.

\section{A. Basic theory of moment principal axes}

The basic idea of block matching is that each frame of the image sequence is divided into many non-overlapping blocks, and the displacement of all pixels in the block is same. Then for every block in the current frame, we make a search for the most similar one within a certain range in the reference frame. The relative offset between the matching block and the current block is the motion vector ${ }^{[18]}$.

\section{B. Block matching criteria}

There are several common best matching criteria: SAD(Sum of Absolute Difference) - MAD(Mean Absolute Difference, MAD) 、 SSE(Sum of Sequence Error) 、 MSE(Mean Sequence Error)、SATD(Sum of Absolute Transformed Difference ) 、 NCCF (Normalized CrossCorrection Function $)^{[19]}$ 。

There are 4 common criteria in block matching algorithm:

1) $\mathrm{NCCF}$

$\operatorname{NCCF}(\mathrm{i}, \mathrm{j})=\frac{\sum_{m=1}^{P} \sum_{n=1}^{P} f_{k}(m, n) f_{k}(m+i, n+j)}{\left(\sum_{m=1}^{P} \sum_{n=1}^{P} f_{k}^{2}(m, n)\right)^{\frac{1}{2}}\left(\sum_{m=1}^{P} \sum_{n=1}^{P} f_{k+1}^{2}(m+i, n+j)\right)^{\frac{1}{2}}}$

2) $M S D$

$\operatorname{MSD}(\mathrm{i}, \mathrm{j})=\frac{1}{P^{2}} \sum_{m=1}^{P} \sum_{n=1}^{P}\left(f_{k}(m, n)-f_{k+1}(m+i, n+j)\right)^{2}$

3) $M A D$

$\operatorname{MAD}(\mathrm{i}, \mathrm{j})=\frac{1}{P^{2}} \sum_{m=1}^{P} \sum_{n=1}^{P}\left|f_{k}(m, n)-f_{k+1}(m+i, n+j)\right|$
4) $S A D$

$\operatorname{SAD}(\mathrm{i}, \mathrm{j})=P^{2} M A D(i, j)=\sum_{m=1}^{P} \sum_{n=1}^{P} \mid f_{k}(m, n)-f_{k+1}(m+$ $i, n+j) \mid(9)$

$\operatorname{SAD}(i, j)=M N^{*}$ MAD $(i, j)$ is usually replaced by the sum of the sum of absolute error MAD.

In the search area, if a sub block which is calculated by the formula above makes square error or absolute difference reach minimal, the block is the matching block to be found. Then the displacement of $(i, j)$ is the matching block motion vector between the current frame and the reference. Matching criterion of matching had little influence on the matching precision, so the above criterions, SAD and MAD, which does not contain multiplication and division become the most commonly used matching criterion.FS (Search Full) matching method is used to search all the sub blocks within the search area and it can get the most matching block in the search area.

\section{Fine image alignment using block matching method}

The search strategy used for block matching is FS(full search ${ }^{[20]}$.What's more, the absolute error function of sad (sum of absolute difference) is used as the matching criterion. Although this search method is accurate, but it is of great computational quantity.In order to improve the program executing speed,we clip the MRI into square image whose size is $48 * 48$, which is the ROI (region of interest). According to the actual situation, we limit the search range: the block size is $4 * 4$, the search range is \pm 8 , namely the search box is in the range of $20 * 20$.

After the three groups of myocardial perfusion images were cut into $48 * 48$ square image, and the SAD block matching method was used for fine alignment. The experimental results are in Table. 2 and Table.3:

Table.2.The alignment results of block matching

\begin{tabular}{|c|c|c|c|}
\hline Group name & I & II & III \\
\hline Reference image & & & \\
\hline $\begin{array}{l}\text { Non-registered } \\
\text { image }\end{array}$ & & & \\
\hline $\begin{array}{l}\text { Registered } \\
\text { image }\end{array}$ & & & \\
\hline $\begin{array}{l}\text { Difference } \\
\text { imagebefore } \\
\text { registration }\end{array}$ & & & \\
\hline \begin{tabular}{l}
\multicolumn{2}{c}{ Difference } \\
image after \\
registration
\end{tabular} & & & \\
\hline
\end{tabular}


Table.3.The alignment results of SAD

\begin{tabular}{|c|c|l|c|}
\hline SAD & $\begin{array}{l}\text { Absolute } \\
\text { error of the non- } \\
\text { alignment and } \\
\text { reference } \\
\text { image/pixel }\end{array}$ & $\begin{array}{l}\text { The } \\
\text { absolute error } \\
\text { of the } \\
\text { registered } \\
\text { image and the } \\
\text { reference } \\
\text { image/pixel }\end{array}$ & $\begin{array}{l}\text { Absolute } \\
\text { error and } \\
\text { reduction } \\
\text { ratio(\%) }\end{array}$ \\
\hline $\mathbf{S A D}_{(\boldsymbol{a})}$ & 44761 & 13441 & $70.0 \%$ \\
\hline $\mathbf{S A D}_{(\boldsymbol{b})}$ & 31599 & 15257 & $51.6 \%$ \\
\hline $\mathbf{S A D}_{(\boldsymbol{c})}$ & 22980 & 11150 & $51.5 \%$ \\
\hline
\end{tabular}

It can be seen that the alignment effect of block matching is satisfactory, and can greatly calibrate the non-rigid deformation of myocardial perfusion images.

\section{CONCLUSIONS}

According to ourexperimental results, the method present in the paper has achieved satisfied aligned effect with fast computational speed. It is demonstrated the combination of principal axes method and block matching method have nice performance for non-rigid alignment of myocardial perfusion image, and can also be extended to other field related to the image alignment.

[1] Jia-bao MO, Nian CAI, Hua-li LI, Liang-ping LUO, Ying WU, Chang-zheng SHI. Diagnostic value of adenosine stress myocardial perfusion SPECT for coronary heart disease : a meta -analysis[J]. Knowledge of cardiovascular disease prevention and control: Academic Edition, 2013 (1): 1-5.(in Chinese)

[2] Wei XU, JianWANG, XinYANG. A GPU Implementation of Compensation Algorithm forCardiac Movement[J].Computer Engineering, 2013, 39(11): 1923, 30.(in Chinese)

[3] Jin-QiangHE, An-PerYE. Alignment of Myocardial Perfusion MRI Images[J].Chinese Journal of Biomedical Engineering. 2005, 24(2): 199-202. (in Chinese)

[4] JianWang,JingweiPan,XinYang. No-rigid alignment for myocardial perfusion MR image[J].Journal of Image and Graphics, 2013, 6: 006. (in Chinese)

[5] Dougherty L, Asmuth J C, Gefter W B. Alignment of CT lung volumes with an optical flow method[J]. Academic radiology, 2003, 10(3): 249-254.

[6] Glocker B, Komodakis N, Tziritas G, et al. Dense image alignment through MRFs and efficient linear programming $[\mathrm{J}]$. Medical image analysis, 2008, 12(6): 731-741.

[7] HuaHUANG, XiaofengZHANG, LuLIU,et a1.Improved sub pixel match approach to cardiac MRI images. Computer Engineering and Applications, 2011, 47(17):162-164. (in Chinese)
[8] Chun-rong WEI, Xiao-fei ZHANG, Hong-bo CHEN, Qiang WANG. A METHOD OF MEDICAL IMAGE ALIGNMENT BASED ON COUROUR EXTRACTION[J].JOURNAL OF GUANGXI NORMAL UNIVERSITY ( Sci\&Tech) , 2003, 21(2): 33-36. (in Chinese)

[9] Guo-xingSONG, JunWEI, Jin-pingLI. Feasibility Research of Principal axes Based Method Used in Coarse Medical Image Alignment[J].JOURNAL OF UNIVERSITY OF JI NAN(ScI\&Tech.), 2008 (4). (in Chinese)

[10] Alpert N M, Bradshaw J F, Kennedy D, et al. The principal axes transformation--a method for image alignment[J]. Journal of nuclear medicine, 1990 (31): 1717-22.

[11] Banerjee P K, Toga A W. Image alignment by integrated rotational and translational transformation matrix[J]. Physics in medicine and biology, 1994, 39(11): 1969.

[12] Slomka P J, Hurwitz G A, Stephenson J, et al. Automated alignment and sizing of myocardial stress and rest scans to three-dimensional normal templates using an image alignment algorithm[J]. Journal of nuclear medicine: official publication, Society of Nuclear Medicine, 1995, 36(6): 1115-1122.

[13] Guoxing Song. Research on alignment and fusion system based on DICOM medical image[D].Jinan University, 2008. DOI:10.7666/d.y1307913. (in Chinese)

[14] Yi Zhang. A Resolution of Electronic Image Stabilization based on block-matching[D]. Harbin Engineering University,2004. (in Chinese)

[15] Gong Xiong. The Research and Realization of Key Techniques of Video Coding Based on H.264/AVC Standard[D].Chong Qin University,2008. (in Chinese)

[16] Min-You WANG, Bin-Jie QIN. Automatic Tumor Detection Based on Block Matching and Geodesic Active Contour[J]. Chinese Journal of Biomedical Engineering, 2008, 26(6): 951-954. (in Chinese)

[17] Qing-kui ZOU, Yi HUANG. Research on algorithm of motion estimation block matching in video compression[J]. Information Technology, 2005, (9):127130. DOI:10.3969/j.issn.1009-2552.2005.09.041. (in Chinese)

[18] You-jun XIANG, Na LEI, Wei-yu YU, Sheng-li XIE. Research of Block Matching Criterion for Motion Estimation[J]. Computer Science, 2009, 36(9):278-280. DOI:10.3969/j.issn.1002-137X.2009.09.072.(in Chinese)

[19] Yue-yuan QIAO, Gui-liang FENG, An-yi LAN. The Research of Block Matching Algorithms for Motion Estimation[J].Journal of Hebei North University(Natual Science Edition), 2006, 22(3): 67-70. (in Chinese)

[20] Chi FENG, Yi ZHANG. Electronic Image Stabilization Based on Block-matching $[\mathrm{J}]$. Automation technology and Application, 2004, 23(6): 49-51. (in Chinese) 